\title{
Stimulated Desorption of Atoms and Molecules from Bodies in the Outer Solar System
}

\author{
R. E. Johnson \\ Engineering Physics \\ Thornton Hall B103 \\ University of Virginia \\ Charlottesville, VA 22903, USA
}

May, 1999

\begin{abstract}
Laboratory data is needed on electronically-induced desorption from low-temperature solids: ices, organics, hydrated salts, glasses and certain minerals. Many bodies in the outer solar system are bombarded by relatively intense fluxes of fast ions and electrons as well as solar UV photons. This can cause both changes in their optical reflectance as well as desorption of atoms and molecules from their surfaces. Stimulated desorption produces $\mathrm{Na}$ and $\mathrm{K}$ 'atmospheres' above the 'rocky' surfaces of the moon and Mercury and $\mathrm{H}_{2} \mathrm{O}, \mathrm{H}_{2}$ and $\mathrm{O}_{2}$ 'atmospheres' about icy outer-solar system bodies. Since theses bodies contain other surface materials, direct detection by spacecraft or remote detection by telescopes of the desorbed atoms and molecules can be used, along with laboratory data, to determine the surface composition and geological processes occurring on distant bodies. This paper describes the relevance of stimulated desorption to the ambient neutrals and plasma in Saturn's magnetosphere, in preparation for CASSINI's arrival, and to the production of atmospheres on the moons of Jupiter being studied by the Galileo spacecraft.
\end{abstract}

\section{Introduction}

An exciting period of exploration of the outer solar system is underway by spacecraft, by remarkably improved ground-based observations and by orbiting telescopes: International Ultraviolet Explorer (IUE) and Hubble Space Telescope (HST). These studies have revolutionized our understanding of the solar system revealing a kaleidoscope of unusual worlds. Because of the low surface temperatures, typically $<130 \mathrm{~K}$, ice is the 'rock' in the outer solar system. That is, excluding the four giant planets and Io, it is the structural and thermal properties of ice that determines the surface geology of many objects from the orbit of Jupiter outward (Burns and Matthews, 1986). Therefore, understanding the radiation chemistry of and desorption from ice or lowtemperature hydrated minerals is critical. Other more volatile molecular species, such as $\mathrm{N}_{2}, \mathrm{O}_{2}, \mathrm{CO}, \mathrm{CO}_{2}$, $\mathrm{NH}_{3}, \mathrm{CH}_{4}$, and $\mathrm{SO}_{2}$ form atmospheres and polar 'ices' or can cause the surface to be geologically active. Io, a moon of Jupiter, is an exception. Owing to its tidal in- teraction with Jupiter, Io is volcanically active and has lost its water and other light volatiles. Because of this, frozen $\mathrm{SO}_{2}$, a volcanic gas on earth, covers Io's surface (Burns and Matthews, 1986).

Since most small, outer solar system bodies, with the exception of Titan, have either no atmospheres or tenuous ones, their icy surfaces are exposed to the solar UV and to the local plasma causing desorption as well as physical and chemical alterations (Johnson, 1990; 1998). During the Voyager I tour of the outer solar system, W.L. Brown, L.J. Lanzerotti and colleagues at AT\&T Bell Labs measured the ejection of molecules induced by energetic ion impact of ice. They discovered that the sputtering from low-temperature ices by fast, light ions is determined by the electronic excitations produced in the ice, rather than by knock-on collisions (Brown et al., 1978) and, hence, is an electronicallystimulated-desorption process. This exciting discovery opened a new field of study. Below the relevance of desorption to a few outer solar system bodies is de- 
scribed; for extended descriptions see Johnson (1990; $1996 ; 1998)$.

\section{Desorption from Solar Sys- tem Bodies}

The samples collected during the Apollo missions show the lunar surface is modified by the impacting solarwind ions $\left(\sim 1 \mathrm{keV} / \mathrm{u} \mathrm{H}^{+}\right.$and $\left.\mathrm{He}^{++}\right)$and by energetic solar particles (Taylor, 1982). This aspect of planetary physics has recently been revived by the observation of Na and K 'atmospheres' around Mercury and the Moon (Potter and Morgan, 1985; 1988). Such atmospheres are produced by stimulated-desorption (the ions, electrons and UV photons) of these atoms from the rocky surfaces (Madey et al. 1998). The sodium atmosphere has been seen to extend to $\sim 5$ lunar radii from the moon's surface (Flynn and Mendillo, 1993), providing an impressive manifestation of desorption.

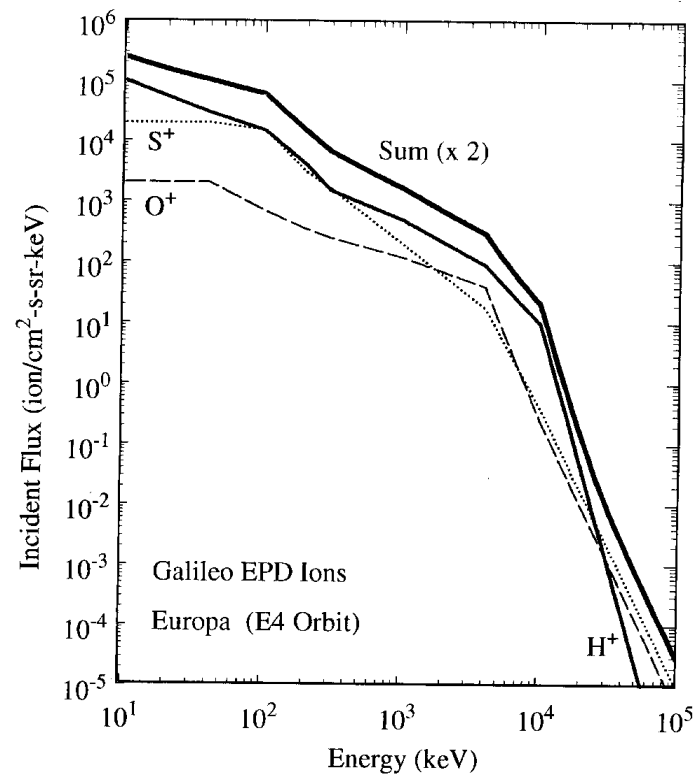

Figure 1. The plasma flux vs. ion energy near Europa a moon of Jupiter. Obtained by the Energetic Particle Detector on the Galileo Voyager spacecraft (e.g., Cooper et al. 1999).

Similarly the ions trapped in the Jovian and Saturnian magnetospheres bombard the surfaces of the embedded moons. These ions are energetic (Fig.1) producing a neutral desorption flux of $\sim 10^{11}$ molecules $/ \mathrm{cm}^{2} / \mathrm{s}$. Although this is much smaller than typical laboratory sputtering rates, the exposure times are long. The application of laboratory data to the erosion of grains has shown that the icy, main rings of Saturn are transient $\left(\sim 10^{8} \mathrm{yrs}\right)$. Of current interest is a ring of micron-sized ice grains called the E-ring, lying outside the main rings in the region where the plasma trapped in Saturn's magnetosphere is relatively intense. Desorption rates based on laboratory data (Shi et al., 1995) place an upper limit on the survival of these charged ice grains (Jurac et al. 1995) of about 1000 years. Therefore, the E-ring must have a source, presumably a relatively recent massive impact or volcanic activity on the moon Enceladus.

Based on laboratory data, desorption from the Ering grains and the surfaces of Saturn's icy moons (Enceladus, Tethys, Dione and Rhea) was predicted to form a very low density but giant toroidal 'atmosphere' of water molecules and water molecule products (Johnson et al. 1989). Remarkably it was detected by Hubble Space Telescope (Shemansky et al., 1993). The desorbed molecules are eventually ionized (Richardson, 1998) supplying fresh ions to the magnetosphere. Because of this, the CASSINI spacecraft is carrying a time-of-flight mass spectrometer. In a close pass of the moon Dione, it will measure the composition of the surface as in a SIMS experiment (Fig. 2).

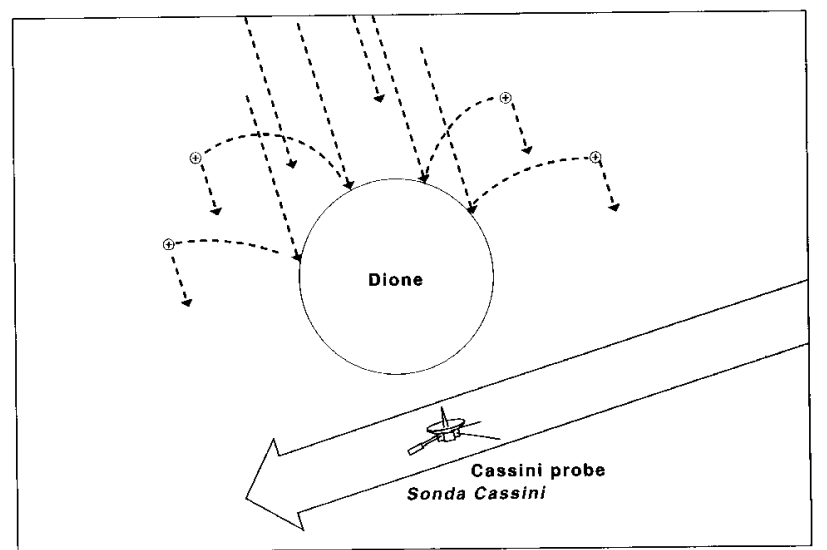

Figure 2. Schematic diagram of the plasma formed from neutrals desorbed from Dione, a moon of Saturn. These will be detected and mass analyzed by the CAPS instrument on Cassini.

Europa, an icy moon of Jupiter, is the subject of intense scrutiny during the extended phase of the Galileo spacecraft mission. This interest is due to the fact that 
it may have a sub-surface ocean and pre-biotic organic materials and hydrated salts on its surface (McCord et al. 1998 a,b; Kargel 1991; Reynolds et al., 1987). In order to confirm these exciting possibilities, the Europa Probe is slated to be the first of NASA's outer solar system (Fire and Ice) missions. Europa also has a thin atmosphere (Hall et al., 1995) with an ionosphere (Kliore et al., 1997) produced by stimulated-desorption of its icy surface by impacting heavy ions from the Jovian plasma torus (Johnson et al. 1982; Saur et al. 1998). Because Europa's surface composition is not known, laboratory data is needed to determine desorption efficiencies from potential surface components. Such data can be used to determine which molecules may populate the atmosphere and may be detectable.

$\mathrm{H}_{2}, \mathrm{O}_{2}$ and $\mathrm{H}_{2} \mathrm{O}$ are the principal species desorbed from an ice. Even for a large moon like Europa most of the $\mathrm{H}_{2}$ escapes directly, but only $\sim 10-25 \%$ of $\mathrm{H}_{2} \mathrm{O}$ and $\sim 5-15 \%$ of the $\mathrm{O}_{2}$ escape (Johnson, 1990). The $\mathrm{H}_{2} \mathrm{O}$ re-condenses on the low temperature surface but the $\mathrm{O}_{2}$ does not. Even at polar temperatures solid $\mathrm{O}_{2}$ would have a significant vapor pressure. Therefore, the $\mathrm{O}_{2}$ molecules produced and desorbed by plasma ion bombardment repeatedly adsorb and thermally desorb until they are dissociated or ionized. Since the loss processes are also determined by the plasma flux, the amount of $\mathrm{O}_{2}$ is self-limiting. The amount of gas-phase $\mathrm{O}_{2}$ was predicted to be very small, $\sim 2 \times 10^{15} \mathrm{~mol} / \mathrm{cm}^{2}$ averaged over the surface (Johnson et al., 1982). Remarkably, such an 'atmosphere' was observed by Hubble Space Telescope (Hall et al., 1995) as stated earlier.

A tenuous atmosphere was also detected in the polar regions of the icy moon Ganymede (Hall et al., 1998) and its 'polar caps' are produced by the plasma alteration of the surface (Johnson, 1997). Recently, a subtle feature in the reflectance spectra of Ganymede's trailing-hemisphere was identified as an absorption band of solid $\mathrm{O}_{2}$ (Calvin et al., 1996). Since the average equatorial surface temperatures of Ganymede exceed those at Europa, the observed $\mathrm{O}_{2}$ is apparently produced and trapped in voids formed in the ice by the plasma irradiation (Johnson and Jesser, 1996). More recently, HST observations revealed that the surface also contains $\mathrm{O}_{3}$ (Noll et al., 1996), which is not a direct radiolytic product of $\mathrm{H}_{2} \mathrm{O}$ but is produced in solid $\mathrm{O}_{2}$ (Gerkaines et al., 1996). Because of the preferential loss of hydrogen, the surfaces are slightly oxidizing (Johnson, 1990; 1998; Johnson and Quickenden, 1997) consistent with the presence of trapped $\mathrm{O}_{2}$ and $\mathrm{O}_{3}$ and the recent observation of $\mathrm{H}_{2} \mathrm{O}_{2}$ in Europa's surface (Carlson et al. 1999). Therefore, $\mathrm{SO}_{2}$, rather than $\mathrm{H}_{2} \mathrm{~S}$ is the dominant species resulting from implantation of $\mathrm{S}$ ions into Europas surface (Johnson et al., 1988).

\section{Organics and Salts}

Studies of the alteration of organic molecules mixed in ices have been made to model the molecular ejecta from comets (e.g., Moore and Tanabe, 1990). In addition, the last twenty years has witnessed a burst of data on molecular desorption by ion bombardment from samples made up of organic molecules, typically, amino acids and proteins (e.g., Johnson and Sundqvist, 1992; Håkansson, 1993). This effort was stimulated by the need to characterized very small samples of organic molecules that are either created synthetically or extracted at various stages when tracking biologic activity. Although laser-pulse-induced desorption is now the preferred laboratory technique, heavy-ion-induced desorption was shown to eject whole molecules, in addition to small volatile molecules produced on decomposition of organics. Therefore, if organic molecules are present on the surface representative molecules will be sputtered into the atmosphere and ionosphere. However, no data is available on energetic ion-induced desorption of organics in an ice matrix.

Whereas heavy ion bombardment of a sample of organic molecules produces both whole molecules and small molecules including $\mathrm{CO}_{2}$, the light ions and UV only produce decomposition with $\mathrm{CO}$ is a primary decomposition product. On an oxidizing surface $\mathrm{CO}$ reacts to become $\mathrm{CO}_{2}$. Therefore, the observations of $\mathrm{CO}_{2}$ in the surface of icy Jovian satellites (McCord et al., 1998a,b) and as a gas-phase species at Callisto (Carlson et al., 1998) could be an indicator of pre-biotic molecules on the surface of these objects. That is, since 
the sputtering of $\mathrm{CO}_{2}$ is more efficient than $\mathrm{H}_{2} \mathrm{O}$, and its destruction and recyling in an icy surface is also efficient, a source is needed. Since $\mathrm{O}_{2}$ comes from decomposition of $\mathrm{H}_{2} \mathrm{O}$, decomposition of organic materials brought to the surface is a likely source of the $\mathrm{CO}_{2}$. If that is the case, other species (whole molecules, HCO, $\mathrm{HCN}$, etc.) should also be present in the atmosphere (Fig.3).

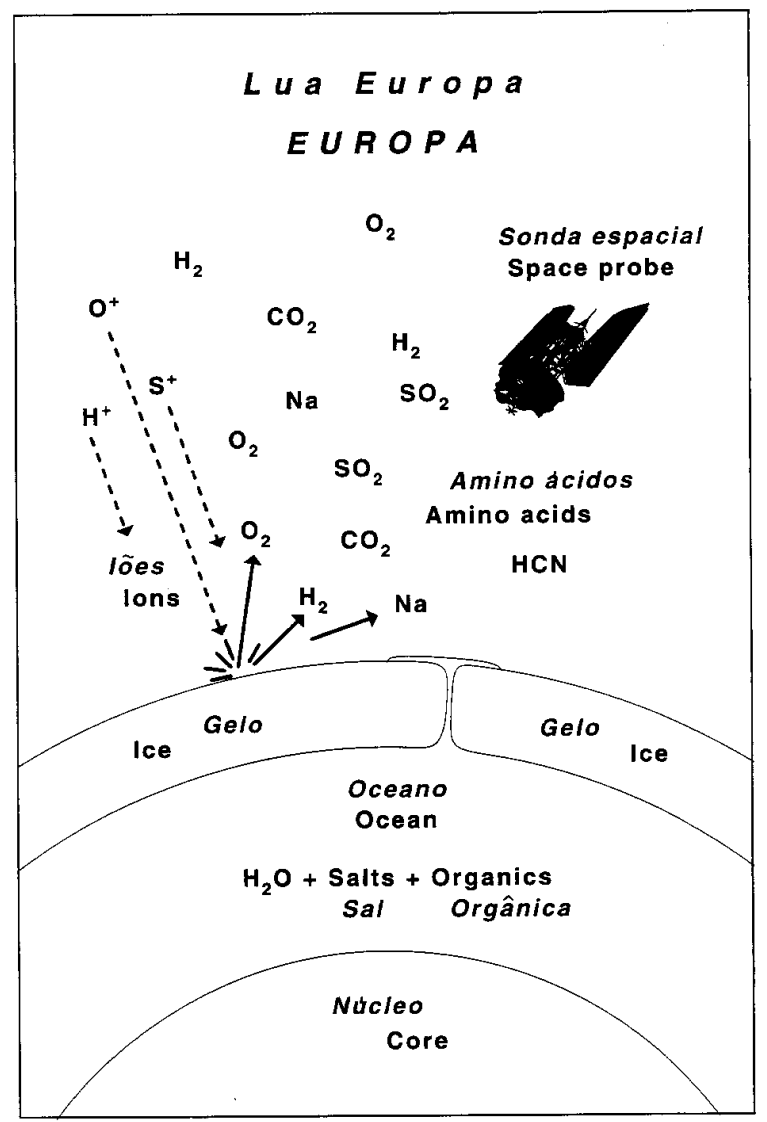

Figure 3. A schematic of the plasma ions in Fig.1 bombarding the surface of Europa producing an ambient gas of desorbed species. These may be representative of an underground ocean containing hydrated salts and organic molecules.

Laboratory studies of the sputtering of salts are less plentiful but key experiments, which can provide guidance, are available. Na has been identified as a trace atmospheric constituent of Europa's atmosphere (Brown and Hill, 1996). Although this could be an implanted species as suggested for sulfur at Europa, $\mathrm{Na}$ is readily desorbed from materials (Madey et al., 1998). $\mathrm{Na}_{2} \mathrm{SO}_{4} \cdot \mathrm{XH}_{2} \mathrm{O}, \mathrm{Na}_{2} \mathrm{CO}_{3} \cdot \mathrm{XH}_{2} \mathrm{O}$ and $\mathrm{MgSO}_{4}$. $\mathrm{XH}_{2} \mathrm{O}$ are materials considered to be indicative of an evaporite from an ocean brine (McCord et al., 1998a; Kargel,
1991). Since the observed $\mathrm{Na}, \mathrm{SO}_{2}$ and $\mathrm{CO}_{2}$ are probably desorption products, it is critical to obtain data on the yields.

If a large moon has a significant gravitationallybound atmosphere, it may be penetrated only by very energetic ions. In this case stimulated-desorption adds trace species to the atmosphere. This occurs at Io, a moon of Jupiter, and, possibly, at Triton, a moon of Neptune. Both Io and Triton have atmospheres supplied by gases venting from the interior and by sublimation of materials more volatile than water ice. At Io, desorption adds the observed $\mathrm{Na}$ to the $\mathrm{SO}_{2}$ atmosphere (Spencer and Schneider, 1995) and penetrating ions modify the sulfur-containing ices, producing darkened polar regions (Johnson, 1997). At Triton, the plasma converts $\mathrm{CH}_{4}$ into $\mathrm{H}_{2}$ plus an organic residue (Thompson et al. 1989) and $\mathrm{NH}_{3}$ into $\mathrm{H}_{2}$ plus $\mathrm{N}_{2}$ (Johnson, 1998). Such processes also may occur at Pluto and its moon Charon (Johnson, 1989).

Reviews of much of the relevant desorption data are available (Johnson, 1990; Johnson and Schou, 1993; Håkansson, 1993; Johnson and Quickenden 1997; Madey et al. 1998; Johnson 1998).

\section{Summary}

Following the discovery that icy objects in the outer solar system are often exposed to a flux of ions and electrons, measurements showed that desorption is driven in ices and other materials by the electronic energy deposited and the desorption yields are large. This data has been applied to describe a toroidal 'atmosphere' and the source of plasma in the Saturnian magnetosphere (Richardson, 1998; Jurac and Johnson, 1999). Based on laboratory measurements that showed that irradiation of a low temperature ice directly produced $\mathrm{O}_{2}$ (Brown et al 1982), the ice-covered moon Europa was predicted to have an extremely tenuous $\mathrm{O}_{2}$ 'atmosphere' (Johnson et al. 1982). Further, the neighboring moon, Ganymede, appears to have $\mathrm{O}_{2}$ and $\mathrm{O}_{3}$ formed and trapped in the radiation-produced defects and voids in its surface (Calvin et al., 1996). 
Since the dominant sputtering agents at Europa are the 100's of keV to MeV O and S ions (Fig.1), the sputtering yields for ice at $\sim 100 \mathrm{~K}$ are large $(\sim 100-1000$ $\mathrm{H}_{2} \mathrm{O}$ /ion; Johnson 1990; Shi et al. 1995). Therefore, trace molecules contained in the ice, such as large organic molecules, may be carried off with the ejecta. Further, other more refractory surface materials, such as the proposed hydrated salts, would also experience decomposition (Johnson et al. 1998), initially losing their water of hydration (Williams, 1998). Therefore, desorbed species other than $\mathrm{O}_{2}$ are present in its atmosphere and ionosphere. In order to determine the surface composition of these interesting bodies, and the possible presence of pre-biotic materials, an intense laboratory program is needed to compliment the spacecraft and HST observations. The Galileo spacecraft is orbiting Jupiter at present and the Cassini spacecraft will soon arrive at Saturn, a probe will be sent to Europa, and the Rossetta mission will put instruments on a comet early in the next century. Therefore, laboratory studies are needed to interpret the expected wealth of data.

\section{Acknowledgement}

This work is supported by the NASA's Planetary Geology and Geophysics Program and by the NSF's Astronomy Program.

\section{References}

Brown, M.E. and R.E. Hill. 1996. Nature 380, 229.

Brown, W.L., L.J. Lanzerotti, J.M. Poate, W.M. Augustyniak, 1978, Phys. Rev. Lett. 49, 1027.

Brown, W.L., W.M. Augustyniak, E. Simmons, K.J. Marcantonio, L.J. Lanzerotti, R.E. Johnson, J.W. Boring, C. T. Reimann, G. Foti, V. Pirronellot, 1982, Nucl. Instrum. Methods 198, 1.

Burns, J.A. and M.K. Matthews, 1986, Satellites (Univ. of Arizona Press, Tucson).

Calvin, W., J.R. Spencer and R.E. Johnson, 1996, Geophys. Res. Lett. 23, 673.

Carlson, R.W., 1998. Science.

Carlson, R.W., et al. 1999 Science in press.
Cooper, J.D., R.E. Johnson, B. Mauk and N. Gerhels. 1999. J. Geophys. Res. Submitted

Flynn, B., and M. Mendillo, 1993, Science 261, 184.

Gerkaines, P.A., W.A. Schutte and Ehrenfreund. 1996. Astron. Astrophys. 312, 289.

Håkansson, P. 1993. In Fundamentals Processes in the Sputtering of Atoms and Molecules (ed. P. Sigmund) (Roy. Dan. Acad., Copenhagen) p. 593.

Hall, D.T., D. Strobel, M. McGrath, M. Feldman, 1995, Nature 373, 677.

Hall, D.T., P.D. Feldman, M.A. McGrath and D.F. Strobel. 1998. Astrophys. J. 499, 475.

Johnson, R.E. 1989. Geophys. Res. Letts 16, 1233

Johnson, R.E., 1990, Energetic Charged-Particle Interactions with Atmospheres and Surfaces (SpringerVerlag, Berlin).

Johnson, R.E. 1996. Rev. Modern Phys. 68, 305.

Johnson, R.E., 1997, Icarus 128, 469.

Johnson, R.E., 1998, Solar System Ices (ed. M. Festou et al.), (Kluwer, Amsterdam), p.303.

Johnson, R.E. and T.I. Quickenden. 1997. J. Geophys. Res. 102, 10985.

Johnson, R.E. and W.A. Jesser. 1997. Astrophys. J. Lett. 480, L79.

Johnson, R.E., and B.U.R. Sundqvist, 1992, Physics Today March, 82.

Johnson, R.E., J. Schou, 1993, in Fundamental Processes in Sputtering of Atoms and Molecules (SPUT 92) ed. P. Sigmund (Roy. Danish Acad., Copenhagen) p.403.

Johnson, R.E., L.J. Lanzerotti, W.L. Brown, 1982, Nucl. Instrum. Methods 198, 147.

Johnson, R.E., M.K. Pospieszalska, E.M.Sieveka, A.F.Cheng, L.J. Lanzerotti and E.C. Sittler. 1989. Icarus 77,311 .

Jurac, S., R.E. Johnson, R.A. Baragiola and B. Donn 1995. J. Geophys. Res. 100, 14, 821.

Jurac, S. and R.E. Johnson. 1999. Icarus submitted.

Kargel, J., 1991, Icarus 94, 368.

Kliore, A.J., D.P. Hinson, F.M. Flasar, A.F. Nagy, and T.E. Cravens. 1997. Science 277, 355.

Madey, T.E., B.V. Yakshinsky, V.N. Ageev and R.E. Johnson. 1998. J. Geophys. Res. 103, 5873 
McCord, T.B. et. al. 1998a. Science 280, 1242.

McCord, T.B. et al. 1998b. J. Geophys. Res. 103, 8603.

Moore, M.H. and T.Tanabe. 1990. Astrophysical J.

Lett. 365, L39.

Noll, K. S., A.L. Lane, R.E. Johnson, and D. Dominque, 1996. Science 273, 341.

Potter, A.E. and T.H. Morgan, 1985, Science 229, 651;

1988, Science 241, 675.

Reynolds, R.T., C.P. McKay and J.F. Kastings.1987

Adv. Space Res. 7, 125

Richardson, J.D., A. Eviatar, M.A. McGrath, and V.M. Vasyliünas 1998, J. Geophys. Res. 103, 20, 245.

Saur, J., D.F. Strobel and F.M. Neubauer, 1998. J.
Geophys. Res. 103, 19947.

Shemansky, D., P. Matheson, D.T. Hall, H.Y. Hu and T.M. Tripp, 1993, Nature 363, 329.

Shi, M., R.A. Baragiola, D.E. Grosjean, R.E. Johnson, S. Jurac and J. Schou, 1995, J. Geophys. Res. 100, 26387.

Spencer, J.A. and N. Schneider, 1996, Ann. Rev. Earth and Planet. Sci. 24, 125.

Taylor, S.R., 1982, Planetary Science: A Lunar Perspective (Lunar and Planetary Inst., Houston).

Thompson, W.R., S.K. Singh, B.N. Khare, and C. Sagan. 1989. Geophys. Res. Lett. 16, 981.

Williams, P. 1998. Private communication. 